\title{
Combating Skill Fade: Basic Life Support refresher training in the remote military environment
}

\section{Inglis $\mathrm{DM}^{1}$, Simmons $\mathbf{G}^{1}$}

1. Institute of Naval Medicine, Royal Navy, Alverstoke, UK.

Introduction: Few interventions are as critical for survival, as the prompt provision of BLS in those patients who have suffered either a cardiac or respiratory arrest. (1) This is especially true in the military environment, where personnel often work in austere and remote locations. The delay in reaching definitive care when deployed, means that prompt near peer BLS is of great importance for ensuring the survival of injured personnel. All deployed Royal Navy (RN) and Royal Fleet Auxiliary (RFA) personnel are, therefore, taught BLS on an annual basis. The phenomenon of skill fade in BLS is widely recognised. (2) This project sought to assess the extent of skill fade via self determined competence and confidence, and to investigate the value of refresher training for personnel deployed overseas on military operations

Methods: The project was undertaken onboard the ship RFA MOUNTS BAY, whilst deployed in the Caribbean on standby to deliver humanitarian relief during the hurricane season. Training was delivered to individuals from the vessel's crew, as well as embarked Royal Navy and British Army personnel. This training was delivered in three sections; a lecture, a practical demonstration, and individual coaching. The training was given to 10 individuals at a time and took 60 minutes to deliver. These sessions were delivered by the ship's Medical Officer (DMI), with additional support from members of The Medical Department. Trainees were given a questionnaire to complete before training asking 1 . Time since last training 2. self reported competence 3. self reported confidence. The participants were asked to answer questions two and three using a ten point Likert scale. On completion of training, the trainees were then asked to answer the same questions again and the results were compared. A "freetext" box was also included to allow for general feedback.

Results: 87 personnel were trained in nine sessions over a timespan of six weeks (approximately half the crew). Each training session followed the same curriculum. Training was delivered using existing equipment (Laerdal ${ }^{\circledR}$ Little Anne ${ }^{\mathrm{TM}}$

\section{Time since last training (months)}

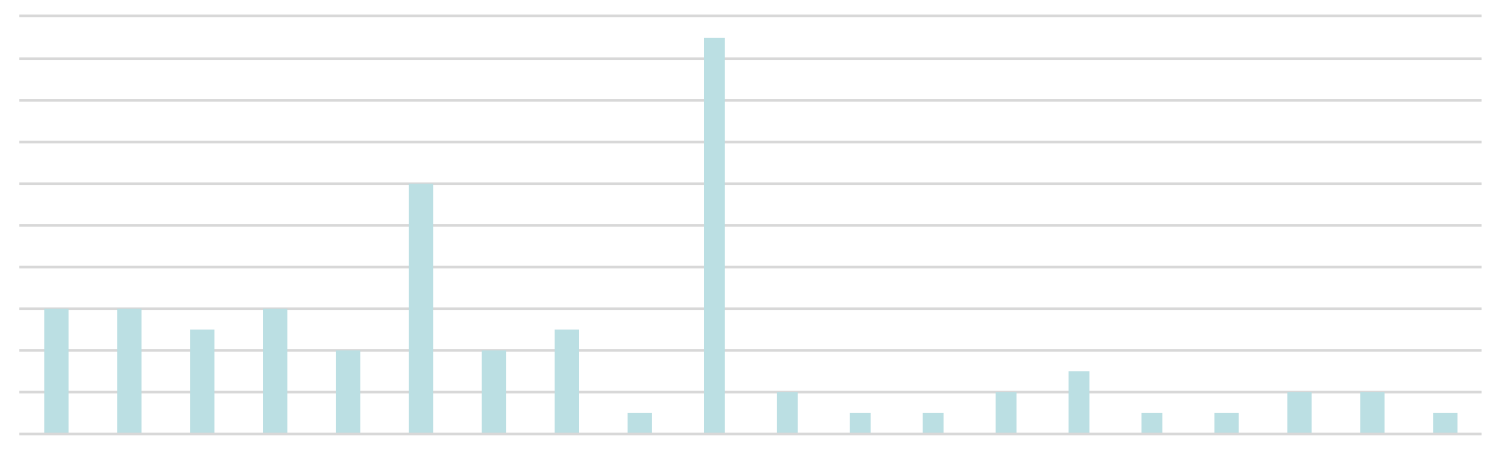

$123 \quad 3 \quad 4 \quad 5 \quad 6 \quad 7 \quad 8 \quad 91214151618242730364855$

Figure 1: Time since last training (months) training manikin). No extra cost was incurred.

The majority of attendees had received formal BLS training within the preceding 12 months; a number of attendees had not had BLS training for significantly longer than this (see Figure 1). Self reported competence and confidence improved following refresher training by $17.3 \%$ and $7.9 \%$ respectively (Figure 2 ). Prior to our refresher training, self-reported competence and confidence was greater if participants had attended training within the preceding 12 months (data not shown). Improvements after our refresher training were greater for those who had not been trained within the last 12 months $-30.0 \%$ and $31.3 \%$ greater improvement in competence and confidence respectively.

46 Always good to do this training. Provides a bit more confidence to people to carry out BLS when needed.

Well worth the refresher course every few months.

Very good. Well presented. A lot of important information. I feel more confident.

Discussion: It is well recognised that skills fade quickly if not routinely practiced - often within 12 months. (2) The majority of participants in this project had been trained within the last 12 months, although a number had not. Refresher training improved BLS confidence and competence and was deliverable at scale with no excess cost. Long deployments away from the UK mean that there is often little time devoted to BLS training. A weakness of this project is that it relies on self reporting to gauge efficacy. Further work is suggested, utilizing an objective measure of BLS competence (Laerdal ${ }^{\circledR}$ SkillReporter ${ }^{\mathrm{TM}}$ ).

Conclusion: We describe here a successful and cost neutral new approach for BLS refresher training, for military personnel deployed in remote locations for extended periods of time. The overall impact of refresher training was modest. Predictably however, the effect was magnified for participants who had not received training recently.

\section{Pre and Post Training Competence and Confidence}

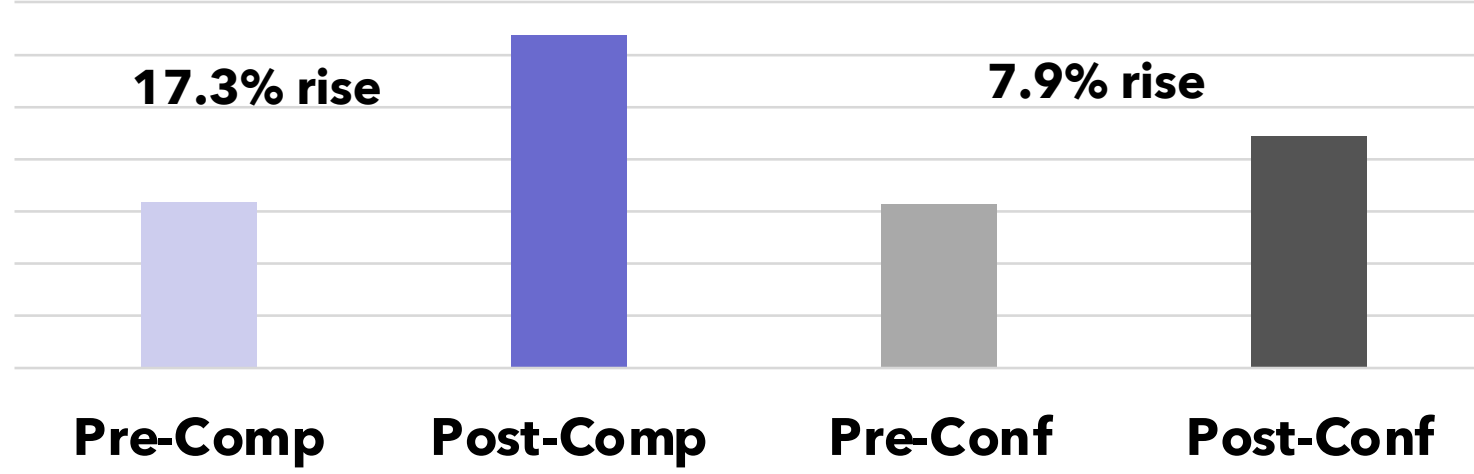

Figure 2: Improvement of competency and confidence following refresher training

\section{References}

(1) Perkins GD, Lockey AS, de Belder MA on behalf of the Community Resuscitation Group, et al. National initiatives to improve outcomes from out-of-hospital cardiac arrest in England. Emerg Med J 2016;33:448-51.

(2) Smith KK, Darlene G, Karen P. Evaluation of staff's retention of ACLS and BLS skills. Resuscitation 2008;78:59-65. 